\title{
Ovarian Seromucinous Tumor
}

National Cancer Institute

\section{Source}

National Cancer Institute. Ovarian Seromucinous Tumor. NCI Thesaurus. Code C4508.

A benign, borderline, or malignant mixed epithelial tumor of the ovary. It is characterized

by the presence of more than one epithelial cell type, most often serous and

endocervical-type mucinous. 